\title{
Efficacy and tolerability of multiple drug therapy in HIV-infected children
}

\author{
Alessandra Viganò $^{a, *}$, Laura Schneider ${ }^{a}$, Vania Giacomet $^{a}$, \\ Roberta Bianchi ${ }^{a}$, Mirko Lo Cicero ${ }^{b}$, Francesca Soster ${ }^{b}$, \\ Elisabetta Bulgheroni ${ }^{\mathrm{b}}$, Stefano Rusconi ${ }^{\mathrm{b}}$
}

\author{
${ }^{a}$ Clinica Pediatrica, L. Sacco Hospital, University of Milan, Via G.B. Grassi 74, 20153 Milano, Italy \\ bIstituto Malattie Infettive e Tropicali, L. Sacco Hospital, University of Milan, Via G.B. Grassi 74, 20153 \\ Milano, Italy
}

Accepted 30 July 2004

Available online 18 September 2004

\section{KEYWORDS \\ Pediatrics; \\ HIV-infection; \\ Multiple drug therapy; \\ Efficacy; \\ Tolerability}

\begin{abstract}
Objectives. To characterize the efficacy and tolerability of multiple drug therapy (MDT) among heavily pre-treated HIV-infected children.

Methods. An observational study of seven children treated with 4-7 antiretroviral agents. MDT regimens were chosen with regard to past antiretroviral exposure and genotypic resistance data. Five children received MDT once, one child twice and one child four times. All patients had AIDS and severe $\mathrm{CD}^{+}{ }^{+}$depletion and failed $>2 \mathrm{PI}$ based HAART regimens.

Results. Virologic response, defined as a $\geq \log _{10}$ decrease in plasma HIV-1 RNA at week 24, was achieved in 7/11 MDT. Successful MDT kept a sustained viral suppression ( $<50$ copies $/ \mathrm{ml}$ ) at longest follow-up (72-96 weeks). Successful MDT obtained a great immune recovery: the median rise in absolute and percentage of $\mathrm{CD}^{+}$cells was 261 and 4 at week 24 and it reached 480 and 16 at 72-96 weeks. Adverse events were common but generally manageable. Mild/moderate gastrointestinal complaints and laboratory abnormalities were detected in 5/11 and 8/11 MDT. Grade 2 severity pancreatitis occurred in one case with chronic active hepatitis C. Pancreatitis resolved within 30 days of MDT interruption.

Conclusions. MDT may be a therapeutic option in children who failed to respond to most standard HAART regimens.

(C) 2004 The British Infection Society. Published by Elsevier Ltd. All rights reserved.
\end{abstract}

\footnotetext{
* Corresponding author. Tel.: +39-02-39042265; fax: +39-025511820.

E-mail address: alessandra.vigano@unimi.it (A. Viganò).
}

\section{Introduction}

Clinical trials and observational studies have shown that highly active antiretroviral treatment (HAART) has dramatically decreased AIDS related mortality and morbidity in HIV-infected adults and children. ${ }^{1,2}$ However, rates of virologic failure, varying from 20 to $50 \%$, have been reported within the first year of 
triple drug therapy in adult patients. Furthermore, rates of virologic failure increase with each successive round of therapy. ${ }^{3}$

In pediatric patients, the virologic response to HAART and the long term control of viral replication seem worse than adult ones. Thus, some studies showed a virologic failure ranging from 20 to $30 \%$, while other studies showed that less than $50 \%$ of children maintained a control of viral replication. ${ }^{4-7}$ These differences observed between children and adults could be associated with one or many of the following: higher baseline plasma HIV-1 RNA levels, previous treatment with mono- or dual-therapy for a long time allowing the presence of highly resistant HIV strains, intolerance to one or more drugs and insufficient drug absorption or poor patients compliance.

Multiple drug therapy (MDT) has been used in adult patients who have failed previous HAART regimens and were not considered to be likely to achieve a long lasting virologic response with standard HAART regimens. In several studies MDT showed an encouraging viral response rate, with 40$50 \%$ patients reaching complete virologic suppression. ${ }^{8-13}$ In addition, a recent study showed that MDT is associated with an improvement on clinical outcome; thus, rates of survival in MDT recipients were comparable with those in patients initiating HAART.$^{14}$ So far, this therapeutic strategy has been assessed in a single pediatric study. King et al. demonstrated that the use of five or more antiretroviral agents or four antiretrovirals plus hydroxyurea in $12 \mathrm{HIV}$-infected children achieved notable virologic response with a tolerable safety profile. ${ }^{15}$

Here, we report the virologic, immunologic and safety outcome of MDT in heavily antiretroviral pretreated HIV-infected children who failed more than two protease inhibitor (PI)-based HAART regimens.

\section{Materials and methods}

\section{Patients and study design}

We included in the study seven HIV-infected children, followed at L. Sacco Hospital, Milan, Italy, who had previously failed at least two separate courses of PI-based HAART regimens. These subjects received 11 courses of MDT regimens consisting of four or more concurrent antiretroviral agents. These regimens were chosen according to patients' history of previous antiretroviral exposure, tolerability and genotypic antiretroviral resistance testing results. The patient's primary physician (A.V.), with the expert advice of the study's virologist (S.R.), determined the specific MDT regimen. Antiretroviral drug doses were calculated for each antiretroviral drug on body weight or surface area and adjusted according to patients' growth.

The efficacy assessment of MDT regimen was planned at 24 weeks. At that time, all patients with virologic response were offered the opportunity to continue the treatment, while patients with virologic failure could discontinue the treatment or start a new MDT regimen according to the results of a new genotypic resistance testing.

Children were included with informed parental consent and the study protocol was approved by $L$. Sacco Hospital Ethical Committee.

\section{Treatment outcome evaluation}

Patients were followed monthly during MDT for clinical outcome, immunologic and virologic responses and development of adverse effects.

Quantitation of HIV-1 RNA was performed by the Roche Amplicor HIV-1 Monitor test, Version 1.0 (Roche Diagnostic Systems, Branchburg, NJ) according to the manufacturer's specifications. Virologic response was defined as $\geq 1 \log _{10}$ decrease in plasma RNA at 24 weeks.

$T$ cells subset was determined from blood samples collected in potassium-EDTA by standard 2-color fluorescent-activated cell sorter analysis using the Coulter EPICS XL Flow Cytometry System (Coulter Electronics, Miami, FL).

Laboratory monitoring for toxicity of antiretrovirals included white blood cell count, platelets, serum creatinine, transaminase, cholesterol, triglycerides, total bilirubin and amylase. Toxicity was defined as a change from laboratory baseline values to a value greater than Grade 2 severity according to the Paediatric Aids Clinical Trials Group toxicity tables. ${ }^{16}$ Patients were questioned at each visit about potential side effects including abdominal pain, peripheral numbness and tingling; all were informed to seek immediate attention if any signs or symptoms occurred.

\section{Adherence}

Before initiating MDT, the goal of complete viral suppression was discussed with families and, when appropriate, with children. The families were provided with written instructions regarding dosages and frequency of administration of prescribed medications. Personal of the HIV team frequently contacted families by telephone after the regimen was started. Adherence to the regimen 
was discussed with the families and, when appropriate, with children at each follow-up visit; difficulties with medications during the past months were sorted out and attempts were made to remove obstacles to compliance.

\section{Resistance testing}

The RNA was extracted by kit QUIAamp HIV (Quiagen, Inc., Chatsworth, CA), according to the manufacturer's recommendation, and converted into cDNA using $10000 \mathrm{HIV}-1$ copies.

The PRO gene was amplified using $10 \mathrm{ml}$ of CDNA and specific primers. Outer primers were P7 and P8. ${ }^{17}$ The second amplification was performed with $10 \mathrm{ml}$ of the first amplification product. The inner primers for the protease region were PRO-F and PRO-R. ${ }^{18}$

Unincorporated primers and nucleotides were removed with QUIAquick spin columns (Quiagen) and PCR products were directly sequenced using internal primers and $A B I$ sequencing kit reagents with dye-labelled dideoxy terminators (Applied Biosystems, Inc., Foster City, CA). A DNA automatic sequencer (ABI PRISM ${ }^{\mathrm{TM}}$ 377, Applied Biosystem, Inc.) was utilized.

Sequences were edited by Factura ${ }^{\mathrm{TM}}$ software and aligned with the HIV-1 IIIB consensus sequence by Sequence Navigator ${ }^{\mathrm{TM}}$ software program (Applied Biosystem).

\section{Results}

\section{Patient demographics}

Demographic, clinical, and antiretroviral treatment features of the seven patients included in the study are shown in Table 1. Subjects (six males/one female) ranged in age from 5.3 to 14.4 years and all of them had a diagnosis of AIDS (CDC clinical category $\mathrm{C}$ ) and severe $\mathrm{CD}^{+}{ }^{+} \mathrm{T}$ cells depletion (CDC immune category 3 ).

The median number of previous antiretrovirals used was 8 (range: 5-13); the median number of previous antiretroviral regimens was 6 (range: 3-9) and the median time of prior exposure to antiretroviral therapy was 84 months (range: 62-114). Among the patients, five were previously treated with NRTI monotherapy, seven with double NRTI therapy, and seven with a median number of 3 (range: 2-5) triple combination therapy. Overall, the patients were previously exposed to a total of 22 HAART regimens, including: 15 combinations with two NRTIs + $1 \mathrm{PI}$, five combinations with one
$\mathrm{NRTI}+1 \mathrm{NNRTI}+1 \mathrm{PI}$, one combination with two NRTIs + 1 NNRTI and one combination with one $\mathrm{NRTI}+2$ PIs. Thus, $100 \%$ of the patients had prior exposure to NRTIs and PIs and $70 \%$ of patients had prior exposure to NNRTIs.

MDT regimens included from 4 to 7 drugs. The great majority of regimens $(8 / 11)$ was composed with one NNRTI and 1-3 NRTIs and PIs, while the other regimens $(3 / 11)$ were composed with 2-3 NRTIs and 2-4 PIs.

All patients received the MDT for 24 weeks at least; the median duration of MDT was 72 weeks (range: 24-96). Five children received one course of MDT regimen and other two children received two (patient 1) and four (patient 7) courses of MDT regimens each. None of the patients had medications delivered by gastrostomy tube and one patient (patient 7) required a naso-gastric tube for the first two courses of MDT.

MDT was permanently discontinued in $2 / 11$ courses: for virologic failure at week 24 in one case and for voluntary cessation by the patient at 72 weeks in the other one.

\section{Analysis of genotypic resistance at baseline}

Genotypic sequences were obtained at 11 timepoints from seven patients before the beginning of any new MDT and in 10/11 cases without interruption of the current antiretroviral treatment.

Primary mutations were found in all cases against the relevant PI that was administered in the last failing regimen. Mutations in the protease gene, related to PI resistance were found in all patients; in particular, L10I was found in four samples, K20R in four samples, D30N in one sample, I54V in 5 samples, and A71V in 6 samples. As far as UPAMs (universal $\mathrm{Pl}$-associated mutations: changes at codons 33, 82, 84, and 90), Fig. 1 indicates a discrete presence of these peculiar mutations in a variable percentage of children at baseline, with the exception of the simultaneous presence of four UPAMs in the same sample. All samples but two presented at least three mutations, with three out 11 sequences showing more than eight codon changes.

The sequence of the RT fragment of pol gene (Fig. 1) showed the presence of M184V mutation in $5 / 11$ samples. All children had a previous history of ZDV treatment, and $\geq 3$ NAMs (nucleoside-associated mutations) were seen in $81.8 \%$ of samples. Some children were treated with d4T, but V75T was never observed. NNRTI-related mutations appeared at a lower frequency, with $\mathrm{K} 103 \mathrm{~N}$ and G190E seen in three samples. 
Table 1 Patients characteristics and summary of antiretroviral regimens

\begin{tabular}{|c|c|c|c|c|c|c|c|c|c|}
\hline Patient & Sex & Age (yrs) & $\begin{array}{l}\text { BMI } \\
\left(\mathrm{kg} / \mathrm{m}^{2}\right)\end{array}$ & $\begin{array}{l}\text { CDC } \\
\text { stage }\end{array}$ & $\begin{array}{l}\text { Prior ARV } \\
\text { exposure }\end{array}$ & $\begin{array}{l}\text { Duration } \\
\text { of prior } \\
\text { ARV } \\
\text { exposure } \\
\text { (mos) }\end{array}$ & $\begin{array}{l}\text { Prior ARV } \\
\text { regimens }\end{array}$ & MDT regimen & $\begin{array}{l}\text { Duration } \\
\text { of MDT } \\
\text { (weeks) }\end{array}$ \\
\hline \multirow[t]{2}{*}{1} & M & 5.3 & 16.1 & $\mathrm{C} 3$ & \multirow[t]{2}{*}{$\begin{array}{l}\text { AZT, 3TC, } \\
\text { ddI, IDV, RTV }\end{array}$} & \multirow[t]{2}{*}{62} & \multirow[t]{2}{*}{5} & $\begin{array}{l}\text { d4T, NVP, } \\
\text { RTV, NFV }\end{array}$ & 24 \\
\hline & M & 7.3 & 16.5 & $\mathrm{C} 3$ & & & & $\begin{array}{l}3 T C, A B C, \\
\text { RTV, SQV, } \\
\text { APV, LPV/RTV }\end{array}$ & 96 \\
\hline 2 & M & 14.3 & 18.5 & $\mathrm{C} 3$ & $\begin{array}{l}\text { AZT, d4T, } \\
3 T C, d d l \\
\text { NVP, IDV }\end{array}$ & 79 & 6 & $\begin{array}{l}\text { ddl, ABC, } \\
\text { APV, IDV }\end{array}$ & 96 \\
\hline 3 & M & 13.6 & 19.1 & $\mathrm{C} 3$ & $\begin{array}{l}\text { AZT, d4T, } \\
3 T C, \text { ddl, } \\
\text { ddC, NVP, } \\
\text { IDV, NFV }\end{array}$ & 84 & 6 & $\begin{array}{l}\text { d4T, ddl, } \\
A B C, E F V, \\
A P V\end{array}$ & 96 \\
\hline 4 & M & 12.9 & 14.7 & $\mathrm{C} 3$ & $\begin{array}{l}\text { AZT, d4T, } \\
3 T C, A B C \\
\text { EFV, IDV, } \\
\text { LPV/RTV }\end{array}$ & 86 & 3 & $\begin{array}{l}\text { ddl, TDF, EFV, } \\
\text { SQV, APV, } \\
\text { RTV }\end{array}$ & 24 \\
\hline 5 & M & 14.4 & 14.1 & $\mathrm{C} 3$ & $\begin{array}{l}\text { AZT, d4T, } \\
3 T C, \text { ddl, } \\
\text { ddC, RTV, } \\
\text { NFV, LPV/RTV }\end{array}$ & 89 & 6 & $\begin{array}{l}\text { TDF, EFV, } \\
\text { APV, LPV/RTV }\end{array}$ & 96 \\
\hline 6 & $\mathrm{~F}$ & 11.2 & 16.0 & $\mathrm{C} 3$ & $\begin{array}{l}\text { AZT, d4T, } \\
3 T C \text {, ddl, } \\
\text { ddC, NVP, } \\
\text { IDV, RTV, NFV }\end{array}$ & 114 & 7 & $\begin{array}{l}\text { ddl, EFV, } \\
\text { SQV, RTV, } \\
\text { LPV/RTV }\end{array}$ & 72 \\
\hline \multirow[t]{4}{*}{7} & M & 7.0 & 12.4 & $\mathrm{C} 3$ & $\begin{array}{l}\text { AZT, d4T, } \\
3 T C, d d l \\
d d C, A B C\end{array}$ & \multirow[t]{4}{*}{81} & \multirow[t]{4}{*}{9} & $\begin{array}{l}\text { d4T, ddl, 3TC, } \\
\text { NVP, SQV, } \\
\text { NFV, RTV }\end{array}$ & 24 \\
\hline & M & 7.5 & 13.3 & $\mathrm{C} 3$ & $\begin{array}{l}\text { NVP, EFV, } \\
\text { IDV, RTV, } \\
\text { NFV, SQV, }\end{array}$ & & & $\begin{array}{l}\text { ddl, d4T, 3TC, } \\
\text { NVP, RTV, } \\
\text { APV, IDV }\end{array}$ & 24 \\
\hline & M & 8.0 & 17.4 & $\mathrm{C} 3$ & APV & & & $\begin{array}{l}\text { d4T, ddl, 3TC, } \\
\text { EFV, SQV, } \\
\text { RTV, LPV/RTV }\end{array}$ & 72 \\
\hline & M & 10.0 & 15.2 & $\mathrm{C} 3$ & & & & $\begin{array}{l}\text { ddI, } 3 T C \text {, } \\
\text { TDF, SQV, } \\
\text { LPV/RTV }\end{array}$ & 96 \\
\hline
\end{tabular}

Note. Yrs, years; BMI, body mass index; ARV, antiretroviral therapy; mos, months; MDT, multiple drug therapy; AZT, zidovudine; d4T, stavudine; ddl, didanosine; 3TC, lamivudine; ABC, abacavir; ddC, zalcitabine; TDF, tenofovir; NVP, nevirapine; EFV, efavirenz; NFV, nelfinavir; RTV, ritonavir; SQV, saquinavir; IDV, indinavir; APV, amprenavir; LPV, lopinavir.

\section{Virologic and immunologic outcomes}

The median plasma HIV-1 RNA at baseline was $5.3 \log _{10}$ copies/ml (range: $4.5-5.7 \log _{10}$ copies $/ \mathrm{ml}$ ) and HIV-1 RNA $>5.0 \log _{10}$ copies $/ \mathrm{ml}$ was detectable in nine cases. The median decrease in plasma HIV RNA 24 weeks after initiation of MDT regimens was $2.0 \log _{10}$ copies/ml (range: 0-3.87 $\log _{10}$ copies $/ \mathrm{ml}$ ). Treatment success, defined as $a \geq 1 \log _{10}$ decrease in plasma HIV-1 RNA at week 24, was achieved in
$7 / 11$ (64\%) MDT courses. In these courses the median HIV-1 RNA value obtained at this point was 909 copies $/ \mathrm{ml}$, ranging from $<50$ to 4753 copies/ml. MDT who achieved a virologic success kept a sustained viral suppression at longer time point. Thus the HIV-1 RNA values available at the longest follow-up (72-96 weeks) were $<50$ copies $/ \mathrm{ml}$ in all cases.

The median absolute and percentage of $\mathrm{CD}^{+}$ cells count prior to the MDT regimens were 147 and 


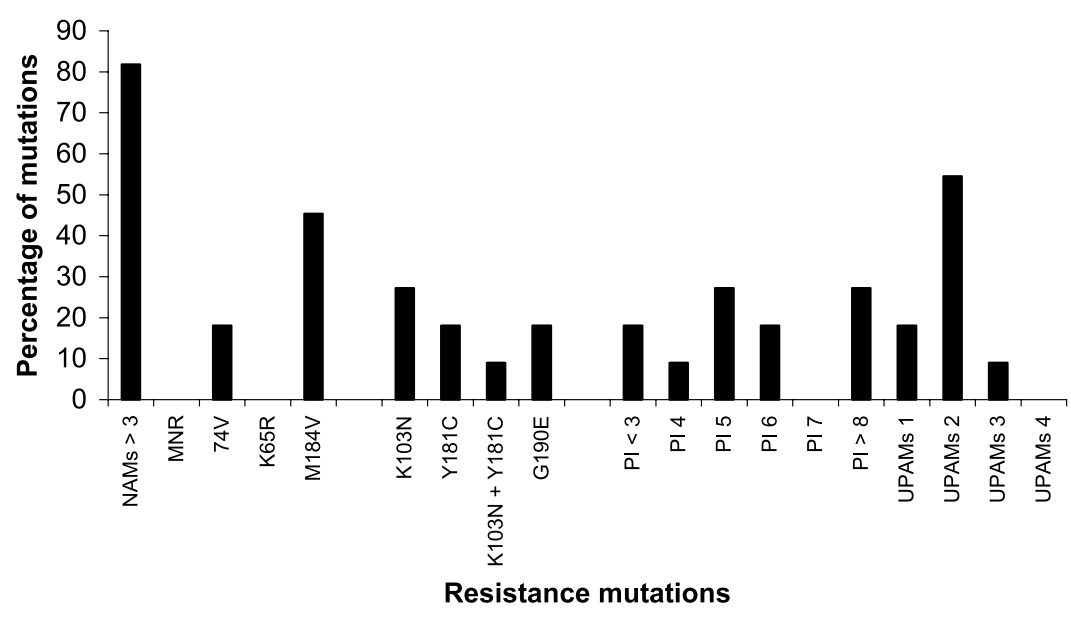

Figure 1 Baseline genotypic resistance profiles. NAMs, nucleoside-associated mutations; MNR, multi-nucleoside resistance (changes at 151-associated codons in the reverse transcriptase region); PI, protease inhibitors; UPAMs, universal PI-associated mutations (changes at codons 32, 82, 84 and 90 in the protease region).

12.6 , respectively. At week 24 , a median increase in absolute and percentage of $\mathrm{CD}^{+}$cells count of 261 (range: -44-433) and 4 (range: 1-15\%) was observed in successful MDT courses. At 72-96 weeks, $\mathrm{CD}^{+}$cells showed a sustained increase with a median rise in absolute and percentage of 480 (range: 459-1171) and 16 (range: 8-22\%) respectively.

\section{Adherence}

Adherence, as assessed through parental reports, was greater than $95 \%$ (missing only one or two doses a month) in $7 / 11$ (64\%) MDT courses. Adherence difficulties have been reported in patient 4 , in patient 1 during his first MDT regimen and in patient 7 during his 2 first MDT regimens. All these MDT regimens did not achieve treatment success at week 24 . Both patient 1 and 7 after instituting a new MDT regimen achieved a virological suppression.

\section{Safety outcomes}

Overall, gastrointestinal complaints occurred in 5/11 (45\%) MDT courses. Four children complained of mild to moderate gastrointestinal symptoms including nausea, vomiting and abdominal pain. Ondansetron was used for nausea prophylaxis in three cases.

Laboratory abnormalities were detected in $5 / 7$ $(71 \%)$ children and these abnormalities occurred in $8 / 11(73 \%)$ MDT courses. In the majority of cases, biochemical and hematologic side effects were not greater than Grade 2 severity according to the Pediatric Aids Clinical Trials Group toxicity tables. ${ }^{16}$ No children developed neutropenia or thrombocytopenia, while moderate anemia (hemoglobin values ranging from 8.5 to $8.9 \mathrm{~g} / \mathrm{dl}$ ) were detected in three children and overall during four courses of MDT. Four children showed Grade 1 hypercholesterolemia with values ranging from 242 to $352 \mathrm{mg} / \mathrm{dl}$. Four children showed Grade 1 hypertriglyceridemia with values ranging from 290 to $544 \mathrm{mg} / \mathrm{dl}$, while one case developed Grade 3 hypertriglyceridemia after 72 weeks of MDT $(1920 \mathrm{mg} / \mathrm{dl})$. This child was treated with gemfibrozil $(1200 \mathrm{mg} /$ day $)$ for 24 weeks with a rapid decrease of triglycerides levels $(296 \mathrm{mg} / \mathrm{dl}$ after 3 months of therapy).

One child (patient 7), with chronic active hepatitis C (moderate piecemeal necrosis, fibrous expansion of portal areas with marked bridging and moderate steatosis) showed increased serum levels of alanine aminotransferase during three out of four courses of MDT. This increase was mild with values ranging from 124 to $167 \mathrm{UI} / \mathrm{L}$. The same patient, at 72 weeks of the third MDT course, showed clinical symptoms consistent with Grade 2 pancreatitis (nausea, vomiting and abdominal pain) associated with an elevation in pancreatic amylase of Grade 4 severity $(2586 \mathrm{UI} / \mathrm{L})$. The patient was receiving a MDT regimen including stavudine and didanosine and gancyclovir for secondary prophylaxis of CMV infection. The symptoms and pancreatic amylase normalized within 30 days of current MDT interruption and a new MDT was restarted.

Lastly, a case of autoimmune thyroiditis was diagnosed in one child (patient 6). This patient had a severe immunodepletion (CD4 ${ }^{+}$count: 7 cells $\left./ \mu \mathrm{l}\right)$ before MDT and showed a wide immunerecovery (CD4 ${ }^{+}$count: 523 cells $/ \mu \mathrm{l}$ ) after 8 months from the beginning of MDT. She presented with marked asthenia and goitre and laboratory data showed 
low FT4 $(6.6 \mathrm{pg} / \mathrm{ml})$ and FT3 $(2.1 \mathrm{pg} / \mathrm{ml})$ values, increased TSH $(44.2 \mu \mathrm{U} / \mathrm{ml})$ and TG $(55 \mathrm{ng} / \mathrm{ml})$ values, positive thyroid autoantibodies (Anti-TSM $123 \mathrm{UI} / \mathrm{ml}$ and anti-TG $666 \mathrm{UI} / \mathrm{ml}$ ).

MDT was not discontinued and L-thyroxine replacement therapy was initiated in this patient with the resolution of symptoms during 6 months of follow-up.

\section{Discussion}

The management of patients who have failed several previous courses of antiretroviral therapy represents a major challenge in clinical practice. Achievement and maintenance of viral load suppression in patients who have not responded virologically to a $\mathrm{PI}$-containing regimen has proved to be relatively uncommon, even more so among triple class experienced patients. ${ }^{19,20}$ We studied a group of heavily pre-treated children who had not only failed to respond to more than $2 \mathrm{PI}$-containing regimens, but also had an earlier AIDS diagnosis and severe $\mathrm{CD} 4^{+}$cells depletion. We adopted a multiple drug rescue therapy regimen consisting of 4-7 drugs on the base of patients' history of previous antiretroviral exposure, tolerability and genotypic resistance testing.

We found a mean viral load decrease of $2.0 \log _{10}$ after 24 weeks of MDT regimen. This change was better than the one observed in an adult cohort treated with 5-9 antiretrovirals $\left(-1.04 \log _{10}\right)$ and similar to the results obtained $\left(2.0 \log _{10}\right)$ in children treated with more than five antiretrovirals or four antiretrovirals plus hydroxyurea. ${ }^{3-15}$ Treatment success, defined as a $\geq 1 \log _{10}$ decrease in plasma HIV-1 RNA at week 24, was achieved in $64 \%$ of MDT and this rate is comparable to what had been reported $(66 \%)$ in the previous pediatrics study. ${ }^{15}$ Moreover, the successful MDT regimens maintained a sustained and prolonged control of viral replication: between weeks 72 and 96 of follow-up HIV-1 RNA was $<50$ copies/ $\mathrm{ml}$ in all cases. These results seem to be better than those obtained in the above mentioned adult and pediatrics cohort (40\% between 47 and 57 weeks and 58\% between 24 and 48 weeks, respectively). ${ }^{3,15}$

Due to the complexities of selecting effective salvage treatment regimens and the high prevalence of drug-resistant virus in previously treated patients, the use of drug-resistance assays to evaluate a patient's virus has a potential clinical benefit for patient management. In addition, recent data showed that therapy interruption not only does not offer any advantage before salvage regimen initiation, but it also could be unsafe. ${ }^{21-23}$

On the base of these observations, almost all MDT regimens were started without interruption of the current antiretroviral regimen. The results of genotypic resistance tests allowed us to avoid the administration of drugs that were indicated as entirely compromised (e.g. the use of any single PI) and to choose favorable combinations. The great majority $(10 / 11)$ of the regimens were composed of a double $\mathrm{Pl}$-containing combination including ritonavir. Virologic success in most patients was probably related to the absence of four UPAMs within the same genotype as well as the favorable pharmacokinetic activity of ritonavir on the regimens including others PIs.

The use of multiple drug regimens seems to achieve a blunting immune reconstitution in HIVinfected adults: some degree of $\mathrm{CD}^{+}$cells count increase has been observed in two studies, while another one showed a lack of $\mathrm{CD}^{+}$cells count improvement. ${ }^{3,9,10}$ The thymic function recovery during potent antiretroviral therapy may allow a better immune reconstitution in pediatric patients. ${ }^{24}$ Thus, an early and great $\mathrm{CD} 4^{+}$cells rise has been observed in naive as well as in NRTIs experienced children following the initiation of HAART. ${ }^{5,25,26}$ In addition, a recent study in NRTIs and PIs experienced children showed that a 3-4 drug regimen obtains at 48 weeks of treatment a mean increase in $\mathrm{CD}^{+}$cells count and percentage of 284 and 5.9 , respectively. ${ }^{27}$ Our data show that successful MDT regimens may obtain an impressive immune recovery even in heavily antiretrovirals pre-treated HIV-infected children with advanced disease. Thus, we observed a median $\mathrm{CD} 4^{+}$cell increase of 261 and 480 cells at 24 and 72-96 weeks of treatment, respectively.

Lack of adherence to the medication regimen has been cited as the primary reason for failure of HAART. Watson et al. found that only $58 \%$ of children were adherent to their medications at level of $75 \%$ or greater. ${ }^{28}$ We adopted a strong support and monitoring of adherence through frequent contacts with children and their families and a careful assessment of parental and child ability and commitment before initiation of MDT regimens. In our study an adherence $\geq 95 \%$ was achieved in $64 \%$ of MDT courses and it was associated with virologic success. Therefore our data suggest that this approach may be useful in improving adherence in children. On the other hand, non-adherence is confirmed to be the primary cause of virologic failure.

Many healthcare workers and HIV-infected individuals are concerned that increased potency 
potentially provided by a MDT might be offset by increased toxic effects. MDT in adult patients is associated with a high frequency $(86 \%)$ of either laboratory abnormalities or subjective adverse drug effects. ${ }^{3}$ In our study adverse drug effects were relatively common and most of them were mild to moderate. Subjective adverse drug effects, involving the gastrointestinal tract, were detected in $45 \%$ of MDT courses. Laboratory abnormalities occurred in the $73 \%$ of MDT courses and alteration in lipid profile was the most frequent abnormality.

Hyperamylasemia seems to be relatively frequent $(6 \%)$ in antiretroviral experienced children treated with a 3-4 drugs regimen and pancreatitis may occasionally occur. ${ }^{27}$ Moreover, an high frequency of hyperamylasemia and pancreatitis has been recently observed in $\mathrm{HCV}$-coinfected patients. ${ }^{29}$ We observed a case of Grade 2 severity pancreatitis in one patient with chronic active hepatitis $\mathrm{C}$ and who was receiving stavudine, didanosine and gancyclovir. Clinical symptoms and hyperamylasemia resolved within 30 days after the interruption of the MDT courses and a new MDT regimen was successfully restarted.

Lastly, an autoimmune thyroiditis was detected in a child with severe immunodepletion who showed a great recovery of $\mathrm{CD}^{+}$cells and it did not interfere with continuation of treatment. In the HAART era a high rate of thyroid autoimmunity and the occurrence of Graves' disease have been reported in deeply immunocompromised adult patients after recovery of high number of $\mathrm{CD}^{+}$ cells. ${ }^{30,31}$ Immune recovery disease may be expected in patients with a favorable response to MDT regimens and specially in children who have an immune reconstitution capacity greater than adults.

In summary, the HIV-infected children treatment with MDT achieve a substantial antiviral response, seems tolerable and it is associated with frequent, but generally manageable, adverse event. These results suggest that MDT deserves further consideration for children who failed to respond to most standard HAART regimens and it will allow to selected patients to eventually benefit from more attractive therapeutic options as they emerge.

\section{Acknowledgements}

This research was supported by a grant no. 30D.84 and 40D.74 from Istituto Superiore di Sanità, Rome, Italy.

\section{References}

1. Palella F, Delaney K, Moorman A, et al. Declining morbidity and mortality among patients with advanced human immunodeficiency virus infection. N Engl J Med 1998;338:853-60.

2. De Martino M, Tovo PA, Balducci $M$, et al. Reduction in mortality with availability of antiretroviral therapy for children with perinatal HIV-1 infection. JAMA 2000;284: 190-7.

3. Montaner JSG, Harrigan PR, Jahnke N, et al. Multiple drug rescue therapy for HIV-infected individuals with prior virologic failure to multiple regimens. AIDS 2001;15(1):61-9.

4. Van Rossum AMC, Niesters HGM, Geelen SPM, et al. Clinical and virologic response to combination treatment with indinavir, zidovudine, and lamivudine in children with human immunodeficiency virus-1 infection: a multicenter study in the Netherlands. J Pediatr 2000;136(6):780-8.

5. Viganò A, Dally L, Bricalli $D$, et al. Clinical and immunovirologic characterization of the efficacy of stavudine, lamivudine, and indinavir in human immunodeficiency virus infection. J Pediatr 1999;135(6):675-82.

6. Nachman SA, Stanley K, Yogev R, et al. Nucleoside analogs plus ritonavir in stable antiretroviral therapy-experienced HIV-infected children. JAMA 2000;283:492-8.

7. Krogstad $P$, Lee $S$, Johnson $G$, et al. Nucleoside-analogue reverse-transcriptase inhibitors plus nevirapine, nelfinavir, or ritonavir for pretreated children infected with human immunodeficiency virus type 1. Clin Infect Dis 2002;34: 991-1001.

8. Montaner JS, Mellors JW. Antiretroviral therapy for previously treated patients. N Engl J Med 2001;345:452-5.

9. Youle $M$, Tyrer $M$, Fisher $M$, et al. Brief report: two-year outcome of a multidrug regimen in patients who did not respond to a protease inhibitor regimen. J Acquir Immune Defic Syndr 2002;29:58-61.

10. Staszewski S, Gute P, Carlebach A, et al. Virological and immunological response to mega-HAART salvage therapy after failure of multiple antiretroviral regimens. AIDS 1998; 12(Suppl. 4):S40.

11. Youle $M$, Mocroft $A$, Johnson $M$, et al. Surrogate marker responses to multidrug combinations comprising hydroxyurea, efavirenz, double protease inhibitors and nucleoside analogues in protease inhibitor failures. Antiviral Ther 1999; 4(Suppl. 1):18-19.

12. Seminari E, Maggiolo $F$, Villani $P$, et al. Efavirenz, nelfinavir and stvaudine rescue combination therapy in HIV-1-positive patients heavily pretreated with nucleoside analogues and protease inhibitors. J Acquir Immune Defic Syndr 1999;22: 453-60.

13. Albrecht MA, Bosch RJ, Hammer $S M$, et al. Nelfinavir, efavirenz, or both after the failure of nucleoside treatment of HIV infection. N Engl J Med 2001;345:398-407.

14. Lee $N$, Hogg RS, Yip B, et al. Rates of disease progression among human immunodeficiency virus-infected persons initiating multiple-drug rescue therapy. J Infect Dis 2003; 188:137-41.

15. King JR, Acosta EP, Chadwick E, et al. Evaluation of multiple drug therapy in human immunodeficiency virus-infected pediatric patients. Pediatr Infect Dis J 2003;22(3):239-44.

16. DAIDS toxicity table for grading severity of pediatric adverse experiences. Available at: http://pactg:cure@roc.s-3.com/ members/download/pedtoxb.doc. Accessed March 28, 2002.

17. Drusano GL, Bilello JA, Stein DS, et al. Factors influencing 
the emergence of resistance to indinavir: role of virologic, immunologic and pharmacologic variables. J Infect Dis 1998; 178:360-7.

18. Rusconi S, La Seta Catamancio S, Citterio $P$, et al. Susceptibility to PNU-140690 (tipranavir) of human immunodeficiency virus type 1 isolates derived from patients with multidrug resistance to other protease inhibitors. Antimicrob Agents Chemother 2000;44:1328-32.

19. Mocroft A, Phillips AN, Miller V, et al. The use of and response to 2 nd-line protease inhibitor regimens: results from the EuroSIDA study. AIDS 2001;15:201-9.

20. Moyle GJ, Wilkins E, Leen C, et al. Salvage therapy with abacavir plus efavirenz or nevirapine in HIV-1-infected persons with previous nucleoside analogue and protease inhibitor use. AIDS 2000;14:1453-4.

21. Lawrence J, Mayers DL, Huppler Hullsiek K, et al. Structured treatment interruptions in patients with multidrug-resistant human immunodeficiency virus. N Engl J Med 2003;349: 837-46.

22. Ruiz L, Ribera E, Bonjoch A, et al. Role of structured treatment interruption before a 5 -drug salvage antiretroviral regimen: the Retrogene study. J Infect Dis 2003;188: 977-85.

23. Deeks SG, Wrin T, Liegler T, et al. Virologic and immunologic consequences of discontinuing combination antiretroviraltherapy in HIV-infected patients with detectable viremia. $N$ Engl J Med 2001;344:472-80.

24. Viganò A, Vella S, Saresella M, et al. Early immune reconstitution after potent antiretroviral therapy in HIVinfected children correlates with the increase in thymus volume. AIDS 2000;14:251-61.

25. Van Rossum AMC, Scherpbier HJ, Van Lochem EG, et al. Therapeutic immune reconstitution in HIV-1-infected children is independent of their age and pretreatment immune status. AIDS 2001;15:2267-75.

26. Gibb DM, Newberry A, Klein N, et al. Immune repopulation after HAART in previously untreated HIV-1-infected children. Lancet 2000;355:1331-2.

27. Sàez-Llorens X, Violari A, Deetz CO, et al. Forty-eight-week evaluation of lopinavir/rotonavir, a new protease inhibitor in human immunodeficiency virus-infected children. Pediatr Infect Dis J 2003;22:216-23.

28. Watson DC, Farley JJ. Efficacy of and adherence to highly active antiretroviral therapy in children infected with human immunodeficiency virus type 1. Pediatr Infect Dis J 1999;18: 682-9.

29. Yoffe B, Bagri AS, Tran T, et al. Hyperlipasemia associated with hepatitis C virus. Dig Dis Sci 2003;48(8):1648-53.

30. Loignon $M$, Martin $M$, Toma $E$. High rate of thyroid autoimmunity and disfunctions in HIV infected adults receiving highly active antiretroviral therapy. Antiviral Ther 2001;6(Suppl. 4):55.

31. Vincent J, Penfornis A, Schillo A, et al. Sequential occurrence of thyroid autoantibodies and Graves disease after immune restoration in severely immunocompromised human immunodeficiency virus-1-infected patients. J Clin Endocrinol Metab 2000;85:4254-7. 